\title{
Use of L-asparaginase in acute lymphoblastic leukemia: recommendations of the Polish Adult Leukemia Group
}

\author{
Beata Piątkowska-Jakubas', Małgorzata Krawczyk-Kuliś², Sebastian Giebel², \\ Maria Adamczyk-Cioch ${ }^{3}$, Anna $\mathrm{Czyż}^{4}$, Ewa Lech-Marańda ${ }^{5}$, Monika Paluszewska $^{6}$, \\ Grażyna Pałynyczko7, Jarosław Piszcz ${ }^{8}$, Jerzy Hołowiecki² \\ 1 Department of Hematology, Jagiellonian University, Medical College, Kraków, Poland \\ 2 Department of Hematology and BMT, Medical University of Silesia, Katowice, Poland \\ 3 Department of Hematology and BMT, Medical University, Lublin, Poland \\ 4 Department of Hematology and Hematopoietic System Proliferative Diseases, Medical University, Poznań, Poland \\ 5 Department of Hematology, Medical University, Łódź, Poland \\ 6 Department of Medicine, Oncology and Hematology, Medical University, Warszawa, Poland \\ 7 Department of Medicine, Institute of Hematology and Transfusiology, Warszawa, Poland \\ 8 Department of Hematology, Medical University, Białystok, Poland
}

\section{KEY WORDS}

antithrombin deficiency, native asparaginase, pegylated asparaginase
Correspondence to:

Beata Piątkowska-Jakubas, MD, $\mathrm{PhD}$, Katedra i Klinika Hematologii, Uniwersytet Jagielloński, Collegium Medicum, 31-501 Kraków, ul. Kopernika 17, Poland, phone: + 48-12-424-76-02 fax: + 48-12-421-96-23, e-mail: bjakubas@wp.pl Received: February 24, 2008. Revision accepted: May 19, 2008. Conflict of interest: none declared. Pol Arch Med Wewn. 2008; 118 (11): 664-669 Translated by Elżiieta Cybulska, MD Copyright by Medycyna Praktyczna, Kraków 2008

\section{ABSTRACT}

L-asparaginase is a hydrolase that catalyzes the conversion of L-asparagine - an endogenous amino acid necessary for the function of some neoplastic cells, such as lymphoblasts. In most human cells deficiency of L-asparagine can be compensated by alternative synthesis pathway through which L-asparagine is produced from aspartic acid and glutamine by asparagine synthethase. Depletion of L-asparagine from plasma by L-asparaginase results in inhibition of RNA and DNA synthesis with the subsequent blastic cell apoptosis. Owing to the unique anti-cancer mechanism of action, L-asparaginase has been introduced to the multi drug chemotherapy in children and adults with acute lymphoblastic leukemia, which has contributed to significant improvement of therapy outcomes and to achieve complete remission in about $90 \%$ of patients. Notwithstanding its high therapeutic efficacy, L-asparaginase can increase the risk of thrombosis. Inhibition of protein synthesis causes most complications observed during treatment with a native and pegylated form of L-asparaginase, including impaired functions of liver, kidneys or central nervous system. Thrombotic events occur as a result of inhibited synthesis of anticoagulant proteins (mainly antithrombin). Coagulopathy has been observed in $1.1-4 \%$ of patients treated with the pegylated L-asparaginase and in $2.1-15 \%$ of those receiving its native form. In this paper approaches to optimize the therapy with L-asparaginase have been discussed.

INTRODUCTION L-asparaginase is an enzyme of the hydrolase class that catalyzes the degradation of L-asparagine - an amino acid (amide of 2-aminosuccinic acid), to aspartic acid and ammonia. This enzyme is present in numerous bacteria, plants and animals. However, it has not been found in humans. The presence of L-asparagine is essential for a normal course of the cell cycle. In most human cells the deficit of the endogenous asparagine may be compensated by alternative synthesis from aspartic and glutamine acid involving the asparagine synthase. This enzyme is absent in certain neoplasmatic cells, particularly in lymphoblasts, thus the depletion of L-asparagine from the plasma leads to DNA, RNA and protein synthesis inhibition and in consequence to blast cell apoptosis. L-asparaginase demonstrates relative substrate specificity and 
at the same time affects the glutamine metabolism, which may intensify adverse effects including hepatotoxicity, hemostatic disorders and hyperglycemia. ${ }^{1}$ Antitumor features of L-asparaginase have been first studied in 1953 by Kidd who observed lymphoma regression in mice and rats in response to the guinea pig plasma. ${ }^{2}$ Further studies have demonstrated that L-asparaginase enzyme accounts for this effect; the mechanism of action of this enzyme has been elucidated. ${ }^{3}$ In the early 70 s L-asparaginase was introduced to combined chemotherapy protocols, mainly in acute lymphoblastic leukemia (ALL) and in non-Hodgkin lymphoma in children, which significantly improved treatment outcomes (93\% remission after induction). ${ }^{4}$

Properties and efficacy L-asparaginase is an important component of induction and consolidation multidrug chemotherapy in children and adults with ALL. The natural (native) forms of the enzyme, produced by Erwinia chrysanthe$m i$ and Escherichia coli bacteria strains are used in treatment, and the E. coli L-asparaginase derivative, covalently bound with mono methoxy polyethylene glycol (Peg-asparaginase, PEG-ASP) of prolonged half-life. The plasma L-asparaginase half-time for the pegylated form is 5.5 days, whereas for the native form it is 26 hours. ${ }^{5}$ Chemotherapy protocols for ALL recommend the dose of $1000-2500 \mathrm{IU} / \mathrm{m}^{2}$ of PEG-ASP, and for the native form $5000-12,000 \mathrm{IU} / \mathrm{m}^{2}{ }^{6}$ There has been published so far one randomized study comparing the properties and clinical efficacy of $E$. coli native and pegylated L-asparaginase in children with newly diagnosed ALL. It demonstrated a more rapid bone marrow blast cell elimination (studied on the 7th and 14th day of induction), L-asparaginase plasma activity time prolongation, and a lower drug immunogenicity in subjects treated with the pegylated form.

In the case of repeated use of the same drug in consolidation, a high titer of anti-L-asparaginase antibodies has been reported in $26 \%$ of patients treated with the native drug and in $2 \%$ of those treated with the pegylated form. The use of PEG-ASP results in maintaining a constant high plasma level of this enzyme with a low titer of antibodies neutralizing its activity with each use. No significant differences were reported regarding toxic symptoms and the risk of infectious complications or the percentage of achieved total complete remissions in both arms. ${ }^{5}$ In earlier published studies on adult patients and children the presence of E.coli L-asparaginase antibodies during multiple administrations was reported in $28-96 \%$ of patients, while severe allergic reactions were observed in $24 \%$ of children and $29 \%$ of adults. ${ }^{7}$

The efficacy of pegylated asparaginase was assessed in studies on children with recurrent ALL, ${ }^{8-10}$ in induction treatment, ${ }^{5,11}$ and in induction in ALL adults. ${ }^{12-14}$ These studies demonstrated a good drug tolerance and a greater efficacy of plasma asparagine elimination in comparison with native L-asparaginase. The influence of the type of L-asparaginase preparation used, and the magnitide of decrease in asparagine plasma levels on treatment outcomes were not assessed in any of these studies.

In the recently published study, Cancer and Leukemia Group B (CALGB) with the use of PEG-ASP in induction treatment and early intensification in ALL adult patients the influence of asparagine elimination on a significantly longer overall survival (OS) and disease free survival (DFS) has been reported for the first time. In patients in whom as a result of pegylated asparaginase administration an efficacious plasma elimination was achieved, and not achieved (defined as the plasma asparaginase level over $0.03 \mathrm{U} / \mathrm{ml}$ lasting for 14 days after administration of minimum 1 of the 4 doses of $2000 \mathrm{U} / \mathrm{m}^{2}$ of PEG-ASP), OS and DFS were 31 and 25 months, and 13 and 12 months, respectively. ${ }^{6}$

Thrombotic complications Treatment of ALL with L-asparaginase may lead to a severe acquired deficiency of serpin class of proteins (inhibitors of serine proteases), mainly antithrombin (AT) and $\alpha \alpha_{1}$-antitrypsin. Studies showed that the prevalence of these complications in children and adults receiving the native drug preparation and the pegylated form is $2.1-15 \%$ and $1.1-4 \%$, respectively. ${ }^{15,16}$

Antithrombin is the main physiologic inhibitor of thrombin and coagulant factors IXa, Xa, XIa. It also antagonizes the activity of factor VII accelerating the dissociation of complexes formed by factor VIIa and tissue factor. Antithrombin forms irreversible links with active enzymes which deprives them of their proteolytic properties. The prevalence of AT deficiency in the population is estimated at $0.02-0.05 \%$. The congenital or acquired AT deficiency is reported in about $2 \%$ of thrombotic patients. Genetic studies have demonstrated a high heterogeneity of the congenital AT deficiency, and on the basis of these studies type I quantitative and type II deficiency (with 3 subtypes of protein qualitative defect) have been distinguished. In type I deficiency (as a result of deletion or point mutation in antithrombin gene that determines AT synthesis) a normal protein synthesis decrease (a reduction in its activity), and the AT antigen level decrease are noted. Qualitative type II is characterized by a defect within the reactive site of the molecule (usually the substitution of a single amino acid) responsible for a decrease in AT inhibitor activity, or a defect of the heparin binding region (so called HBS heparin binding site mutation). In qualitative deficiency subtype III there are multiple abnormalities in the reactive site, the heparin binding site and a decreased AT plasma level. All type I and II deficiencies are inherited in an autosomal dominant manner, with the exception of the heparin binding region defect, inherited as an autosomal recessive trait. 
Acquired antithrombin deficiency may result from defective synthesis (liver diseases, protein losing enteropathy), an increased consumption during acute thrombosis, intravascular coagulation syndrome with fibrinolysis activation, sepsis, hemolytic post-transfusion reaction, nephrotic syndrome (protein loss) and as the result of heparin, L-asparaginase, and oral contraceptives and estrogen treatment (a negligible level decrease).

The influence of L-asparaginase on the level, activity and conformation of plasma AT molecules in ALL patients was assessed, and ultrastructure alterations were studied in mouse hepatic cells arising from L-asparaginase intraperitoneal injections, and in vitro in HepG2 cell lines (human hepatoma) incubated with L-asparaginase. It has been reported that with L-asparaginase there is an antithrombin molecule conformation change and stability loss, and as a consequence, formation of protein aggregates which accumulate in the endoplasmatic reticulum cisterns. In effect there is antithrombin secretion impairment as in its genetically determined type I deficiency. Conformation alterations, as in the case of other serpins (serine proteases inhibiting proteins) induce a loss of plasticity of AT molecules which determines its proper function. L-asparaginase alters conformation and enhances intracellular accumulation of formed AT molecules. They are regarded as the main mechanism responsible for thrombotic complications in patients treated with L-asparaginase preparations. ${ }^{17}$

It has also been demonstrated that despite a significantly decreased fibrinogen level there may be L-asparaginase treatment induced thrombosis, which results mainly from the synthesis inhibition, not from consumption in the process of thrombosis. In the group of 93 ALL patients analyzed, there were 9 in 93 (10\%) thrombotic episodes including 6 in 93 (7\%) with L-asparaginase treatment. A decrease in plasma fibrinogen levels below $50 \mathrm{mg} / \mathrm{dl}$ reported in 12 in 93 cases (13\%) significantly correlated with the risk of thrombotic events. Several investigators showed that a significant reduction in fibrinogen levels $(<50 \mathrm{mg} / \mathrm{dl})$ in L-asparaginase-treated patients reflects the degree of protein synthesis inhibition, including AT, which leads to the conclusion that measurements of fibrinogen levels may serve as a useful and easily accessible indicator of the risk of thrombosis occurrence in this group of patients. ${ }^{18}$

Some authors recommend antithrombin substitution when its plasma level decreases below $60 \%$ of normal values during $L$-asparaginase treatment, aiming at decreasing the thrombotic risk.

In the studies GIMEMA (Gruppo Italiano Malattie Ematologiche Maligne dell'Adulto) by an Italian group including adult patients, the following AT dosage scheme is recommended for prophylaxis: $20-25$ and $50 \mathrm{IU} / \mathrm{kg}$ of body mass for 7-10 consecutive days, beginning on the day of the native L-asparaginase treatment start (a dose of $6000 \mathrm{IU} / \mathrm{m}^{2}$ s.c. for 7 days). ${ }^{19,20}$ During AT prophylactic administration, coagulopathy syndromes, and proteins $C$ and $S$, plasminogen, $\alpha \alpha_{2}$-antiplasmin, factor VII and platelet count alterations were not observed. ${ }^{20}$

Subsequent studies have confirmed the efficacy of such management during L-asparaginase treatment in adults: thrombotic complications occurred only in patients not receiving AT prophylaxis ( $27 \%$ vs. $0 \%$ in the prophylaxis group) ${ }^{21}$

The only randomized controlled trial published so far, PARKAA (Prophylactic Antithrombin Replacement in Kids with Acute Lymphoblastic Leukemia Treated with Asparaginase), that assessed the safety and efficacy of AT prophylactic administration in children treated with L-asparaginase, demonstrated a tendency towards a smaller percentage of thrombi in the group treated with AT (28\%) in comparison with the group not receiving AT (37\%). To diagnose thrombosis, phlebography, vein ultrasound, echocardiography and magnetic resonance examinations have been performed. ${ }^{22,24}$

The most recent reports published by pediatric groups indicate the role of combined prophylaxis with low-molecular-weight heparin (enoxaparin $\mathrm{AT}$ at the dose of $1 \mathrm{mg} / \mathrm{kg} /$ day) and AT in thrombotic complications prophylaxis in children (lack of thrombotic complications in the group receiving combined prophylaxis with enoxaparin and AT vs. $12.7 \%$ in the group receiving AT as prophylaxis). ${ }^{23}$

The analysis of congenital and acquired hemostasis disorders which may increase the risk of thrombotic events in children treated with L-asparaginase demonstrated a prothrombotic tendency in those with antiphospholipid antibodies. ${ }^{23}$ This suggests the purposefulness of excluding the antiphospholipid syndrome, deficiency of congenital and acquired coagulation inhibitors and resistance to activated $C$ protein, before introducing asparaginase treatment in patients with a history of thrombosis.

In case of suspected inherited thrombophilia, indications for this therapy should be considered on the individual basis. Patients who after administration of PEG-ASP had thrombotic or hemorrhagic complications should not receive native L-asparaginase.

Adverse effects related to protein synthesis inhibition The majority of adverse effects during L-asparaginase treatment are related with a transient protein inhibition in the liver and pancreas. Increased activity of hepatic enzymes, including aminotransferases, alkaline phosphatase, and increased serum billirubin levels occur in 5-16\% of patients with PEG-ASP administration (for 14 days) and in nearly $100 \%$ patients after native $\mathrm{L}$-asparaginase treatment. Acute pancreatitis is one of the most severe complications commonly occurring without a significant increase in, or with normal enzyme levels, pancreatic amylase and lipase (the effect of their synthesis inhibition 
by L-asparaginase). It occurs in $1-5.7 \%$ of patients treated with the pegylated form and in about $10 \%$ of patients receiving the native form. Concomitant clinical symptoms are not characteristic (nausea, vomiting, and abdominal pain). In the majority of cases the inflammation is subclinical and resolves with the drug activity cessation. Total parenteral nutrition and decompression stomach tube insertion are recommended in management. L-asparaginase induced pancreatitis in single cases may have a fulminant hemorrhagic or necrotic course and has a fatal prognosis. ${ }^{16} \mathrm{Sin}-$ gle case reports, mainly in children, documented an efficacious therapy with the use of percutaneous drainage and pancreas site rinsing in necrotic pancreatitis. ${ }^{25}$ Patients with previous acute pancreatitis cannot be treated with L-asparaginase preparations.

Insulin synthesis inhibition, glutamine metabolism disorders and simultaneous use of corticosteroids in induction, described in the Introduction, may induce hyperosmolar hyperglycemia (after administrating PEG-ASP in $2-3 \%$ and with the use of the native form in about $4 \%$ of patients).${ }^{16}$ Frequent glycemia monitoring on insulin therapy is recommended in order to avoid rapid hypoglycemia induced by normalized endogenous insulin synthesis after discontinuation of L-asparaginase activity.

Central nervous system symptoms Central nervous system symptoms (CNS), i.e. somnolence, amentia and hallucinations, are reported in $33 \%$ of patients treated with PEG-ASP and in $25 \%$ of patients during treatment with the native form. Serious consciousness disturbances, like stupor and coma, are described in case reports. The clinical presentation requires differentiation with symptoms of CNS leukemic invasion, and ischemic or hemorrhagic lesion. The plasma ammonia accumulation (hydrolysis product) and low L-asparagine and/or L-glutamine levels in the cerebral tissue are probably the basis for these lesions. These symptoms resolve spontaneously when the levels of these substances are back to normal. ${ }^{26}$ This situation requires follow-up and corrections of possible acid-base disorders.

SUMIMARY 1 It is considered that the immunogenicity of the asparaginase preparation plays a key role in maintaining the therapeutic plasma level of the enzyme. With multiple use of L-asparaginase its activity is inhibited as a result of antibody formation. This justifies the use of the pegylated form in induction (of low immunogenicity), and the native E.coli L-asparaginase preparation in consolidation.

2 Thrombotic risk during L-asparaginase treatment can be reduced by prophylaxis with AT preparations at the dose of 25-50 IU/kg from the day of treatment initiation through the whole period of the drug activity. Achieving an AT plasma activity of $80-100 \%$ is desirable. For the native form used in the PALG algorithm the recommended
AT prophylaxis time is about 10 days, for PEG-ASP (a single dose, activity time about 5.5 days), the period of administration is estimated individually with hemostatic and AT system monitoring. If AT preparations cannot be administered, fresh frozen plasma preparations may be used as a substitute.

3 Patients with thromboembolic disease should not receive L-asparaginase treatment.

4 L-asparaginase administration requires coagulation system and biochemical parameter monitoring including hepatic enzymes, pancreatic amylase, glycemia, and acid-base balance.

\section{REFERENCES}

1 Müller HJ, Boos J. Use of L-asparaginase in childhood ALL. Crit Rev Oncol Hematol. 1998; 28: 97-113.

2 Kidd JG. Regression of transplanted lymphomas induced in vivo by means of normal guinea pig serum. I. Course of transplanted cancers of various kinds in mice and rats given guinea pig serum, horse serum, or rabbit serum. J Exp Med. 1953; 98: 565-582.

3 Broome JD. L-Asparaginase: discovery and development as a tumor-inhibitory agent. Cancer Treat Rep. 1981; 65: 111-114.

4 Ortega JA, Nesbit ME Jr, Donaldson MH. L-Asparaginase, vincristine and prednisone for induction of first remission in acute lymphocytic leukemia. Cancer Res. 1977; 37: 535-540.

5 Avramis VI, Sencer S, Periclou AP, et al. A randomized comparison of native Escherichia coli asparaginase and polyethylene glycol conjugated asparaginase for treatment of children with newly diagnosed standard-risk acute lymphoblastic leukemia: a Children's Cancer Group study. Blood. 2002; 99: 1986-1994.

6 Wetzler M, Sanford BL, Kurtzberg J, et al. Effective asparagine depletion with pegylated asparaginase results in improved outcomes in adult acute lymphoblastic leukemia: Cancer and Leukemia Group B Study 9511. Blood. 2007; 109: 4164-4167.

7 Woo MH, Hak LJ, Storm MC, et al. Hypersensitivity or development of antibodies to asparaginase does not impact treatment outcome of childhood acute lymphoblastic leukemia. J Clin Oncol. 2000; 18: 1525-1532.

8 Ettinger LJ, Kurtzberg J, Voute PA, et al. An open-label, multicenter study of polyethylene glycol-L-asparaginase for the treatment of acute lymphoblastic leukemia. Cancer. 1995; 75: 1176-1181.

9 Müller HJ, Löning L, Horn A, et al. Pegylated asparaginase (Oncaspar) in children with ALL: drug monitoring in reinduction according to the ALL/NHL-BFM 95 protocols. Br J Haematol. 2000; 110: 379-384.

10 Jarrar M, Gaynon PS, Periclou AP, et al. Asparagine depletion after pegylated $\mathrm{E}$. coli asparaginase treatment and induction outcome in children with acute lymphoblastic leukemia in first bone marrow relapse: $A$ Children's Oncology Group study (CCG-1941). Pediatr Blood Cancer. 2006; 47 $141-146$.

11 Rizzari C, Citterio M, Zucchetti M, et al. A pharmacological study on pegylated asparaginase used in front-line treatment of children with acute lymphoblastic leukemia. Haematologica. 2006; 91: 24-31.

12 Rosen 0 , Muller $\mathrm{HJ}$, Gokbuget $\mathrm{N}$, et al. Pegylated asparaginase in combination with high-dose methotrexate for consolidation in adult acute lymphoblastic leukaemia in first remission: a pilot study. Br J Haematol. 2003; 123: $836-841$

13 Douer D, Yampolsky H, Cohen LJ, et al. Pharmacodynamics and safety of intravenous pegaspargase during remission induction in adults aged 55 years or younger with newly diagnosed acute lymphoblastic leukemia. Blood. 2007; 109: 2744-2750.

14. Hołowiecki J, Giebel S, Krawczyk M et al. PEG-Asparaginase as a Part of Induction Therapy for Adult Acute Lymphoblastic Leukemia. A Phase II Study by the Polish Adult Leukemia Group (PALG). 45-th Annual Meeting of ASH, 6-9.12.2003 San Diego, USA. Blood. 2003; 102: 255b.

15 Nowak-Göttl U, Ahlke E, Fleischhack G, et al. Thromboembolic events in children with acute lymphoblastic leukemia (BFM protocols): prednisone versus dexamethasone administration. Blood. 2003; 101: 2529-2533.

16 Holle LM. Pegaspargase: an alternative? Ann Pharmacother. 1997; 31 : 616-623.

17 Hernandez-Espinoza D, Minano A, Martinez C, et al. L-Asparaginase-induced antithrombin type I deficiency. Am J Pathol. 2006; 169: 142-153.

18 Beinart G, Damon L. Thrombosis associated with L-asparaginase therapy and low fibrinogen levels in adult acute lymphoblastic leukemia. Am J Hematol. 2004; 77: 331-335

19 Mattioli Belmonte M, Gugliotta L, Delvos U, et al. A regimen for antithrombin III substitution in patients with acute lymphoblastic leukemia under treatment with L-asparaginase. Haematologica. 1991; 76: 209-214. 
20 Mazzucconi MG, Gugliotta L, Leone G, et al. Antithrombin III infusion suppresses the hypercoagulable state in adult acute lymphoblastic leukaemia patients treated with a low dose of Escherichia coli L-asparaginase. A GIMEMA study. Blood Coagul Fibrinolysis. 1994; 5: 23-28.

21 Elliott MA, Wolf RC, Hook CC, et al. Thromboembolism in adults with acute lymphoblastic leukemia during induction with L-asparaginase-containing multi-agent regimens: incidence, risk factors, and possible role of antithrombin. Leuk Lymph. 2004; 45: 1545-1549.

22 Mitchell L, Andrew M, Hanna K, et al. Trend to efficacy and safety using antithrombin concentrate in prevention of thrombosis in children receiving L-asparaginase for acute lymphoblastic leukemia. Results of the PAARKA study. Thromb Haemost. 2003; 90: 235-244.

23 Meister B, Kropshofer G, Klein-Franke A, et al. Comparison of low-molecular-weight heparin and antithrombin versus antithrombin alone for the prevention of symptomatic venous thromboembolism in children with acute lymphoblastic leukemia. Pediatr Blood Cancer. 2008; 50: 298-303

24 Mitchell LG, Andrew M, Hanna K, et al. A prospective cohort study determining the prevalence of thrombotic events in children with acute lymphoblastic leukemia and a central venous line who are treated with L-asparaginase: results of the Prophylactic Antithrombin Replacement in Kids with Acute Lymphoblastic Leukemia Treated with Asparaginase (PARKAA) Study. Cancer. 2003; 97: 508-516.

25 Top PC, Tissing WJ, Kuiper JW, et al. L-asparaginase-induced severe necrotizing pancreatitis successfully treated with percutaneous drainage. Pediatr Blood Cancer. 2005; 44: 95-97.

26 Chabner BA, Loo TL. Enzyme therapy: L-asparaginase. In: Chabner BA Longo DL (eds). Cancer chemotherapy and biotherapy: principles and practice. Lippincott-Raven, Philadelphia. 1996: 485-492. 